\title{
Reducing complication rates and hospital readmissions while revising the enhanced recovery after bariatric surgery (ERABS) protocol
}

\author{
Marjolijn Leeman $^{1}$ (i) $\cdot$ Stefanie R. van Mil ${ }^{1} \cdot$ L. Ulas Biter $^{1} \cdot$ Jan A. Apers ${ }^{1} \cdot$ Kees Verhoef $^{2} \cdot$ Martin Dunkelgrun $^{1}$
}

Received: 21 May 2019 / Accepted: 10 February 2020

(C) Springer Science+Business Media, LLC, part of Springer Nature 2020

\begin{abstract}
Background To optimize the postoperative phase following bariatric surgery, the enhanced recovery after bariatric surgery pathway (ERABS) has been developed. The aim of ERABS is to create a care path that is as safe, efficient and patient-friendly as possible. Continuous evaluation and optimization of ERABS are important to ensure a safe treatment path and may result in better outcomes. The objective of this study was to compare the clinical outcomes of patients undergoing bariatric surgery over 2014-2017, during which the ERABS protocol was continuously evaluated and optimized.

Methods This is a retrospective cohort study. Data were collected from patients undergoing a primary Roux-en-Y gastric bypass or sleeve gastrectomy between January 2014 and December 2017. Outcomes were early complications, unplanned hospital revisits, readmissions, duration of surgery and length of hospital stay.

Results 2889 patients underwent a primary bariatric procedure in a single center. There was a significant decrease in minor complications over the years from 7.0 to $1.9 \%(p<0.001)$. Hospital revisit rates decreased after $2015(p<0.001)$. Readmission rates decreased over time $(p<0.001)$. The mean duration of surgery decreased from 52 (in 2014) to 41 (in 2017) minutes $(p<0.001)$. Median length of hospital stay decreased from 1.8 to 1.5 days in $2015(p=0.002)$ and remained stable since.

Conclusion An improvement of the ERABS protocol was associated with a decrease in minor complication rates, number of unplanned hospital revisits and readmission rates after primary bariatric procedures.
\end{abstract}

Keywords Bariatric surgery $\cdot$ Fast-track $\cdot$ Enhanced recovery after surgery $\cdot$ ERABS $\cdot$ Gastric bypass $\cdot$ Gastric sleeve

Obesity has become pandemic over the past decades [1]. The obesity-related comorbidities, mortality and costs emphasize the need for both adequate prevention and treatment strategies. Bariatric surgery is the only long-term effective treatment for morbid obesity, with better results in terms of weight loss and resolution of obesity-associated comorbidities in comparison to non-surgical interventions [2].

At the end of the twentieth century, the enhanced recovery after surgery (ERAS) program was introduced for colorectal surgery [3] to standardize perioperative care and thereby provide more efficient, safe and cost-effective care. Subsequently, several study groups described an ERASlike program for bariatric surgery implemented within their

Marjolijn Leeman

M.Leeman@Franciscus.nl

1 Department of Surgery, Franciscus Gasthuis \& Vlietland, Kleiweg 500, 3045 PM Rotterdam, The Netherlands

2 Department of Surgery, Erasmus Medical Center, Rotterdam, The Netherlands own clinics [4-7]. These publications eventually lead to the composition of an official enhanced recovery after bariatric surgery (ERABS) program by the ERAS Society in 2016, setting the standard for and leading to the implementation of ERABS on a worldwide scale [8].

A meta-analysis of published studies on ERABS programs demonstrated the benefits of ERABS, such as a decreased length of hospital stay (LOS) without an increase of complications or readmissions [9]. This could lead to more efficient and cost-effective bariatric care. After the implementation of the ERABS program in 2012 within our own clinic, the number of unplanned revisits to the outpatient clinic or emergency ward and the readmission rate was significantly increased from 12.5 to $16.8 \%$, without an increase in the incidence of severe complications. Most patients, who revisited the hospital shortly after discharge, had complaints of persisting pain or nausea, while serious complications were ruled out. The hypothesized reason for this was that patients were insufficiently informed on the postoperative course, when leaving the hospital [7]. To complement our ERABS protocol with the most up-to-date 
evidence-based and experience-based knowledge, the pathway is continuously under evaluation and improved where possible.

The aim of this study was to evaluate the outcomes of patients undergoing bariatric surgery between 2014 and 2017. In this period, the ERABS protocol was continuously being evaluated and optimized. Primary outcome measure was deviation from standard postoperative course, expressed as early complications, hospital readmissions and returns to emergency department or unscheduled visits to the outpatient clinic within 30 days postoperatively. Secondary outcome measures were duration of surgery and LOS.

\section{Materials and methods}

\section{Design and setting}

This was a retrospective cohort study with prospective data collection in the period between 2014 and 2017 in a singlecenter setting. The Franciscus \& Vlietland Hospital in Rotterdam, the Netherlands has a bariatric clinic mainly performing laparoscopic Roux-en-Y gastric bypasses (LRYGB) and laparoscopic gastric sleeve gastrectomies (LSG). Since 2014 there has been an increase in patients undergoing a mini gastric bypass-one anastomosis gastric bypass (MGBOAGB) or revisional surgery. All patients were treated according to the ERABS program [7]. The patients were divided into groups based on the year of surgery.

\section{Data collection}

Data were collected from the electronic patient files of all consecutive patients undergoing a primary bariatric LRYGB or LSG in the period of January 2014 until December 2017. Patients undergoing a MGB-OAGB $(n=145)$ or revisional surgery $(n=228)$ were excluded, due to the relatively small numbers of procedures.

\section{Outcomes}

Outcome measures were (1) early complications, (2) readmissions and (3) returns to the emergency department or unscheduled visits to the surgical outpatient clinic within 30 days postoperative. Complications were defined as minor or major complications, based on the guidelines described by Brethauer et al. [10].

\section{The revised ERABS protocol of the Franciscus Hospital}

The ERABS protocol was implemented in the Franciscus Hospital in the course of 2012. The protocol was composed by a multidisciplinary team with delegates from all involved departments and was based on the guidelines published by
Fried et al. [11]. Patients are referred to the bariatric center by their general practitioner and are evaluated for surgery according to the IFSO criteria [11]. Following the IFSO guidelines, patients up to the age of 65 are candidates for surgery [8]. All patients undergoing a bariatric procedure are treated according to the ERABS protocol and the protocol is the same for all bariatric procedure types. Next to several recommendations from the guidelines that were adopted in the protocol, additional alterations were made to the ERABS protocol itself. The latest ERABS protocol is described in the next paragraphs and summarized in Table 1. The protocol consists of a preoperative phase, perioperative phase and postoperative phase.

\section{Preoperative phase}

On the intake day, patients are initially screened by the bariatric nurse on BMI and comorbidities. After confirmation of the patient meeting the (IFSO) criteria, the patient is screened by a dietician and a psychologist.

On the analysis day, on average about 8 weeks later, an endocrinologist screens the patient in combination with a physical examination, looking for genetic or pathologic causes of obesity. A dietician evaluates the patients' compliance to their dietary advices to predict the chance of postoperative complications due to the patients eating behavior. In case of concerns about eligibility for bariatric surgery by the surgeon, physician, dietician or psychologist, patients are discussed in a weekly multidisciplinary meeting.

On the planning day, on average about 2 weeks later, the patient is screened by the surgeon and the type of surgery is chosen (RYGB, SG or MGB-OAGB). An anesthesiologist screens the patient at the preoperative screening unit and trains the patient to self-administer subcutaneous low molecular weight heparin (LMWH) if indicated. The waiting list for bariatric procedures is about 8 weeks.

\section{Perioperative phase}

Patients are admitted on the day of surgery and can eat solid food up to $6 \mathrm{~h}$ before surgery and clear fluids up to two hours before surgery. Patients receive anti-embolism stockings only when indicated: in case of earlier thromboembolic events or other risk factors. Patients are instructed to urinate just before departure to the OR to avoid the need for urinary catheters.

Patients do not receive sedative premedication in the holding bay. Patients receive $3 \mathrm{~g}$ of cefazolin or, in case of allergies, $600 \mathrm{mg}$ clindamycin. For analgesia, $1000 \mathrm{mg}$ acetaminophen intravenous is used and patients receive $4 \mathrm{mg}$ of dexamethasone and $4 \mathrm{mg}$ ondansetron as prophylactic anti-emetics. The patient is positioned while awake to avoid decubitus during surgery. The anesthesia protocol 
Table 1 Key points of the ERABS protocol in the Franciscus Hospital

Information evening: extensive provision of information with films and interviews
Intake day: screening by bariatric nurse, dietician and psychologist
Analysis day: screening by physician, dietician and if indicated psychologist
Planning day: screening by surgeon and anesthesiologist
Mandatory weighing 1 week prior to surgery and at admission on the day of surgery
Start LMWH (Dalteparin 5000 IE) on the evening before surgery
Anti-thrombosis stockings in case of DVT or PE
Intake of solid food up to 6 h and clear fluids up to 2 h prior to surgery
No urinary catheters
No sedative premedication
Scheduling of high-risk patients first on the OR
Antibiotics, analgesia and anti-emetics 15 min before surgery
Patient in French position with anti-Trendelenburg, head positioned on special HELP cushion
Early ambulation by asking patient to slide into their bed from the operation table
Direct encouraging to drink full liquid diet and ambulate
Analgesia with 4 times daily 1000 mg acetaminophen and 2 times daily 10 mg oxycodone when necessary
Decrease anti-diabetic medication immediately for drug-dependent T2DM with close monitoring
Low administration of intravenous fluids, decreased in accordance to oral intake
Extra group session with dietician on the morning of discharge
Mobilizing under guidance of physical therapist
Discharge when patient meets discharge criteria

has undergone some minimal changes. Induction is done with $100 \mathrm{mcg}$ remifentanil, combined with propofol titrated to effect (200-300 $\mathrm{mg}$ ) and rocuronium bromide 30-40 mg. Using a Head Elevated Laryngoscopy Position (HELP) cushion, intubation is done by the anesthesiologist. While the surgery is performed, the patient receives remifentanil 10-30 $\mathrm{ml} / \mathrm{h}$, desflurane, 10-15 mg morphine and $10-15 \mathrm{mg}$ ketamine. The operation is performed using intra-abdominal pressure up to $20 \mathrm{mmHg}$, to warrant good surgical overview and working space in the obese patient. For termination, remifentanil and desflurane are discontinued and sugammadex $100 \mathrm{mg}$ is administered. As soon as the patient wakes, the patient slides by themselves from the operating table onto a bed and is taken to the PACU. There, extra analgesia is only administered if indicated.

Patients are encouraged to mobilize as soon as they return from the OR. During admission the patient receives Dalteparin 5000 IE subcutaneously. Standardized pain protocol includes four times daily $1000 \mathrm{mg}$ acetaminophen intravenous and-only if needed-up to six times daily 10-15 mg morphine intramuscular, for maximally $24 \mathrm{~h}$. The usage of non-steroidal anti-inflammatory drugs (NSAIDs) was discouraged. The day after surgery, a standardized checklist is filled in by the ward doctor during morning rounds. A physical therapist helps the patient with mobilization and gives instructions and tips to take home. Intravenous fluid administration is quickly reduced to zero when liquid intake is sufficient. Patients are discharged in case of no suspicion of postoperative complications.

\section{Protocol alterations}

Based on the finding that patients were returning to the outpatient clinic or emergency ward more often, due to insufficient knowledge on the postoperative course and not due to major complications, the described protocol has undergone several alterations. Firstly, in 2014, a postoperative bariatric checklist was implemented to evaluate the safety of early discharge [12]. Based on predetermined parameters and cut-off points, a decision was made on the patient's discharge. Interestingly, the patient's willingness to leave the hospital was one of the significant predictors of presence or development of major complications. The checklist has become standard care within our ERABS program since 2014.

Secondly, as of 2016, the role of the dietician, psychologist and physical therapist grew importance. A psychologist already screened all patients on the intake day and can guide patients throughout the perioperative phase with additional consulting if needed. A physical therapist no longer screens patients preoperatively, but helps with early mobilization of patients on the first day postoperatively and provides information on what to expect in the postoperative period. In addition to the preoperative counseling by a dietician, an extra group lecture is held on the first postoperative day, in which patients are reminded of the content of the diet and 
importance of compliance to this diet. We believe that the best strategy to inform patients on the postoperative course is spreading out the education over multiple visits. Therefore, during each preoperative visit, all caregivers spend time informing the patient on their own area of expertise.

The hypothesis is that the patient gains confidence in recovering at home after practicing mobilization under the guidance of the physical therapist and having refreshed the information on dietary habits.

\section{Statistical analysis}

All analyses were performed using SPSS (PASW) 18.0 software (SPSS Inc., Chicago, Illinois, USA). Multivariable binary logistic regression was used to estimate the relationship between year of surgery and clinical outcome, correcting for age, gender, BMI at inclusion, hypertension, diabetes, dyslipidemia and type of surgery. Multivariate analysis was used to evaluate the differences in minor and major complication rates between the different types of procedures, corrected for surgeon, baseline characteristics and type of procedure. Multivariate analysis was also used for comparing the percentages of patients revisiting the hospital without having a complication over the years, correcting for the same covariates. Results were evaluated at a significance threshold of $p<0.05$ (two-sided).

\section{Results}

Between January 2014 and December 20172889 patients underwent a primary LRYGB or LSG within the Franciscus Hospital. Table 2 shows the patient characteristics and specifications of the procedures. No differences were found in baseline characteristics between the cohorts. The number of bariatric procedures that were performed by the different surgeons in 2014 varied from sixteen to 359 LRYGBs and fourteen to 417 LSGs, illustrating the wide range in surgical experience between the surgeons.

Figure 1 shows the complication rates over the years since the introduction of the ERABS program. There was a significant decline in the rate of overall complications occurring within 30 days between 2014 and $2017(p<0.001)$. Especially the minor complications decreased dramatically from $7.0 \%$ in 2014 to $1.9 \%$ in 2017 ( $p<0.001$ ). The major complication rate was $4 \%$ on average over the years and did not change significantly $(p=0.467)$. There were no significant differences in minor complication rates $(p=0.144)$ or major complication rates $(p=0.932)$ between LRYGB and LSG. Table 3 shows that the year of surgery significantly influenced minor complication rates $(p=0.002)$, but not major complication rates $(p=0.552)$, when using multivariable analysis, correcting for type of surgery, gender, age, BMI and comorbidities. Table 3 also shows that the surgeon did

Table 2 Patient characteristics

\begin{tabular}{lllll}
\hline Characteristics & $\begin{array}{l}2014 \\
(n=669)\end{array}$ & $\begin{array}{l}2015 \\
(n=598)\end{array}$ & $\begin{array}{l}2016 \\
(n=847)\end{array}$ & $\begin{array}{l}2017 \\
(n=775)\end{array}$ \\
\hline Age at surgery (years) (median, IQR) & $44(34.5-51.1)$ & $43(33.6-50.5)$ & $43(32.4-50.3)$ & $43.2(33.0-51.3)$ \\
Female gender (\%) & 79.4 & 79.9 & 82.1 & 81.9 \\
BMI at inclusion $\left(\mathrm{kg} / \mathrm{m}^{2}\right)$ (mean, SD) & $43.7(5.4)$ & $43.7(4.8)$ & $43.3(4.7)$ & $42.6(4.6)$ \\
Hypertension (\%) & 22.3 & 32.9 & 23.6 & 28.0 \\
Diabetes (\%) & 15.4 & 19.7 & 16.6 & 11.9 \\
Dyslipidemia (\%) & 19.0 & 18.4 & 13.7 & 12.8 \\
Roux-en-Y gastric bypass (\%) & 55.8 & 65.1 & 61.3 & 46.7 \\
\hline
\end{tabular}

Fig. 1 Crude overall complication rates between 2014 and 2016. There was a significant decrease in 2017 compared with 2014 ( $p<0.001)$, mainly due to the decrease in minor complications $(p<0.001)$. The major complication rate did not change over the years $(p=0.467)$

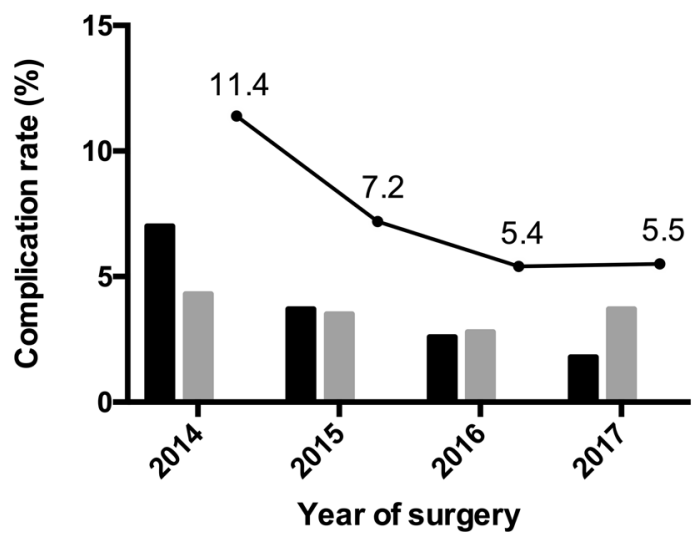


not influence minor complication rates $(p=0.582)$ or major complication rates $(p=0.885)$ significantly. Mortality within 30 days has remained stable with on average $0.05 \%$ each year.

Figure 2 shows the rate of unplanned revisits to the outpatient clinic or emergency department within 30 days postoperatively. There was a significant increase in hospital revisits between 2014 and 2015 from 18 to 22\%, without an increase in complications (Fig. 1). Since then, the amount of hospital revisits has gradually decreased to $14 \%$ and was significantly lower in 2017 compared to $2015(p<0.001)$. The percentage of patients revisiting the hospital without having a complication was increased to $18 \%$ in 2016 , but later fell to $10 \%$ in 2017.

Figure 3 shows that the rate of hospital readmissions within 30 days postoperative significantly decreased over the years $(p<0.001)$. Especially the percentage of patients being readmitted in the hospital without any (major) complications was minimal in $2017(1 \%)$, making a bigger percentage of the readmissions justified. There were no significant differences in readmission rates between LRYGB and LSG $(p=0.278)$. Also, there were no significant differences among the surgeons in minor complication rates $(p=0.774)$, major complication rates $(p=0.901)$ or readmission rates $(p=0.950)$.

Figure 4 shows the decrease in total duration of surgery, including anesthesiological care, from 73 (in 2014) to 60 (in 2017) minutes in the OR $(p<0.001)$. A similar trend was seen regarding the decrease in duration of surgery from 52 (in 2014) to 41 (in 2017) minutes.

Figure 5 displays the decrease in LOS from median 1.8 to 1.5 days in $2015(p=0.002)$ and remained stable ever since.
Table 3 Multivariate analysis of year of surgery and surgeon on complication rates
Fig. 2 Percentage of hospital revisits within 30 days postoperative $(p<0.001)$ and crude percentage of patients with and without major complications

\begin{tabular}{|c|c|c|c|c|c|c|}
\hline & \multicolumn{2}{|c|}{ Minor complication rates } & \multicolumn{2}{|c|}{ Major complication rates } & \multicolumn{2}{|c|}{ Any complication rates } \\
\hline & OR $95 \% \mathrm{CI}$ & Sig & OR $95 \% \mathrm{CI}$ & Sig & OR 95\% CI & Sig \\
\hline Year of surgery & & 0.002 & & 0.552 & & 0.005 \\
\hline 2015 vs. 2014 & $\begin{array}{r}0.588 \\
(0.327-1.058)\end{array}$ & 0.076 & $\begin{array}{r}0.787 \\
(0.420-1.475)\end{array}$ & 0.455 & $\begin{array}{r}0.654 \\
(0.422-1.013)\end{array}$ & 0.057 \\
\hline 2016 vs. 2014 & $\begin{array}{r}0.439 \\
(0.247-0.778)\end{array}$ & 0.005 & $\begin{array}{r}0.641 \\
(0.351-1.171)\end{array}$ & 0.148 & $\begin{array}{r}0.507 \\
(0.333-0.774)\end{array}$ & 0.002 \\
\hline 2017 vs. 2014 & $\begin{array}{r}0.314 \\
(0.162-0.607)\end{array}$ & 0.001 & $\begin{array}{r}0.818 \\
(0.462-1.449)\end{array}$ & 0.491 & $\begin{array}{r}0.524 \\
(0.342-0.804)\end{array}$ & 0.003 \\
\hline Surgeon & & 0.582 & & 0.885 & & 0.888 \\
\hline Surgeon 1 vs. 5 & $\begin{array}{r}0.592 \\
(0.292-1.202)\end{array}$ & 0.147 & $\begin{array}{r}1.380 \\
(0.459-4.154)\end{array}$ & 0.567 & $\begin{array}{r}0.766 \\
(0.419-1.399)\end{array}$ & 0.386 \\
\hline Surgeon 2 vs. 5 & $\begin{array}{r}0.648 \\
(0.300-1.403)\end{array}$ & 0.271 & $\begin{array}{r}1.664 \\
(0.533-5.195)\end{array}$ & 0.381 & $\begin{array}{r}0.885 \\
(0.467-1.679)\end{array}$ & 0.709 \\
\hline Surgeon 3 vs. 5 & $\begin{array}{r}0.514 \\
(0.197-1.342)\end{array}$ & 0.174 & $\begin{array}{r}1.586 \\
(0.459-5.478)\end{array}$ & 0.465 & $\begin{array}{r}0.776 \\
(0.369-1.632)\end{array}$ & 0.504 \\
\hline Surgeon 4 vs. 5 & $\begin{array}{r}0.526 \\
(0.235-1.177)\end{array}$ & 0.118 & $\begin{array}{r}1.623 \\
(0.516-5.100)\end{array}$ & 0.407 & $\begin{array}{r}0.785 \\
(0.409-1.507)\end{array}$ & 0.467 \\
\hline
\end{tabular}

Data were corrected for type of surgery, gender, age, BMI and comorbidities

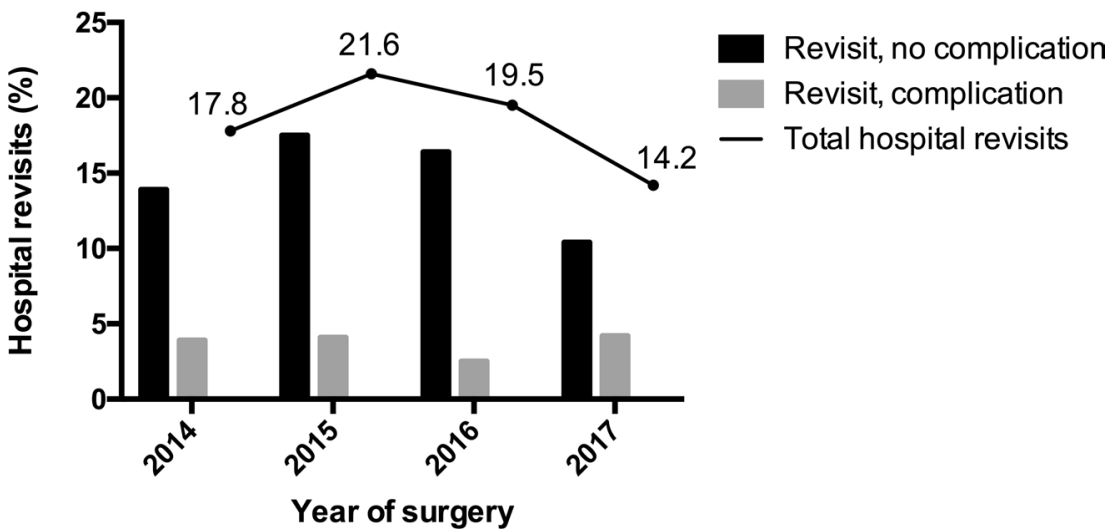


Fig. 3 Crude hospital readmission rates within 30 days, decreasing over the years when comparing 2014 and 2017 $(p<0.001)$

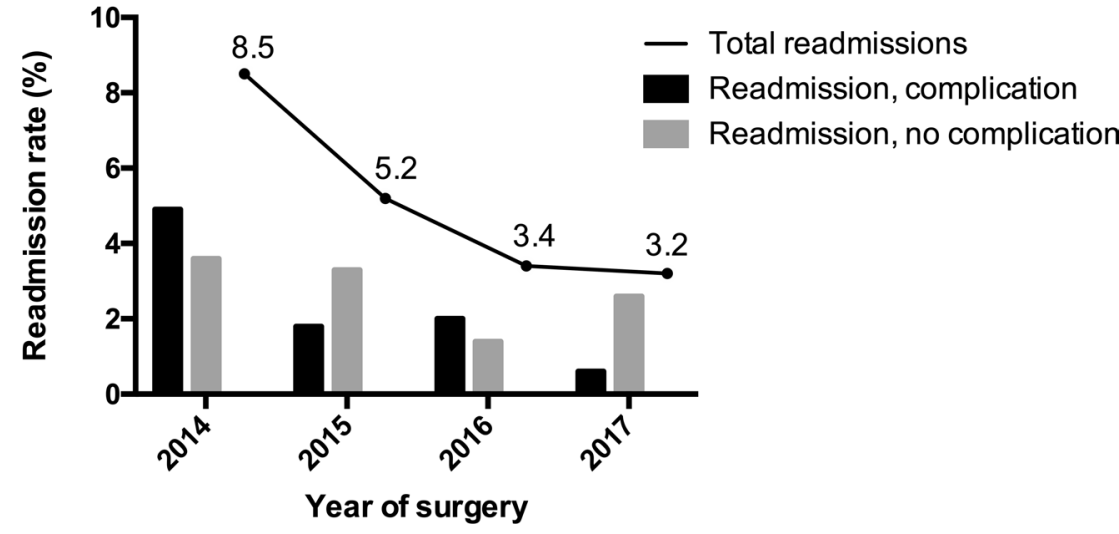

Fig. 4 Crude mean length of procedure in minutes, divided in perioperative time and length of surgery $(p<0.001)$

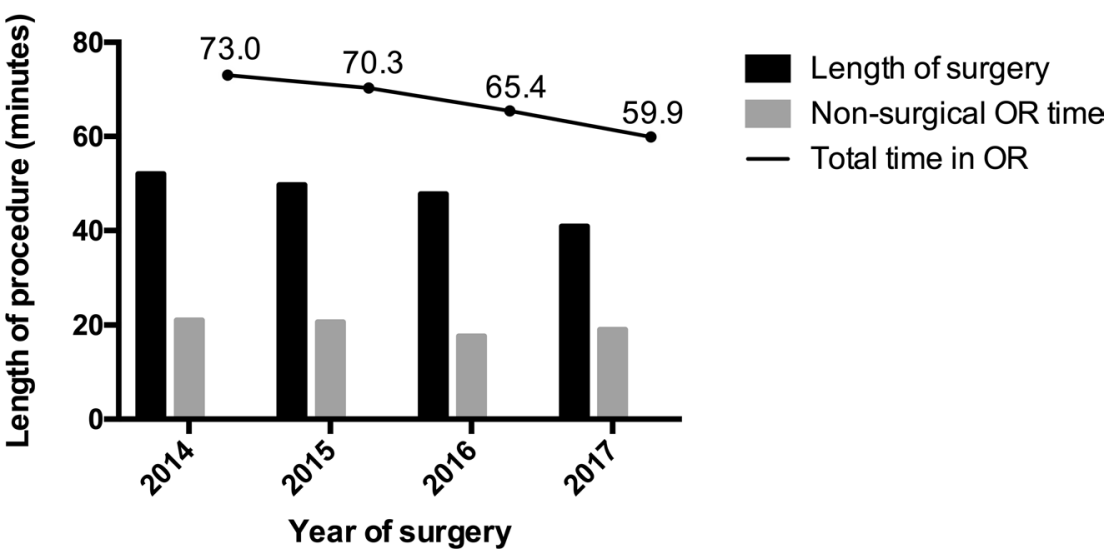

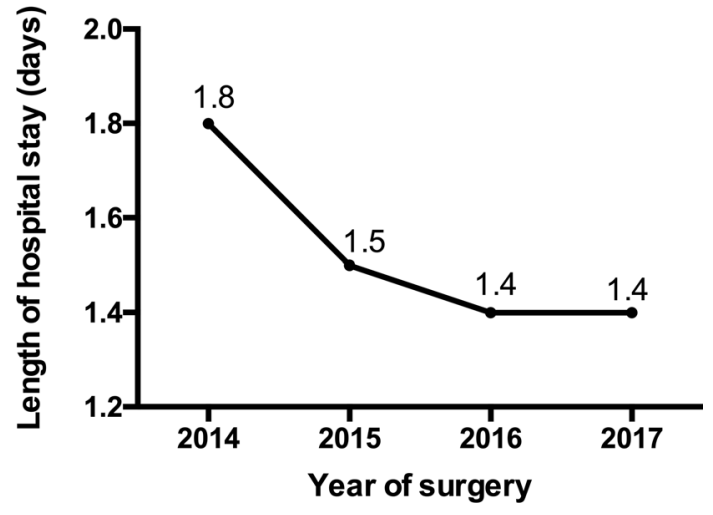

Fig. 5 Crude mean length of hospital stay, stabilizing since 2015. There was a significant decrease in LOS from 2014 to 2015 $(p=0.048)$

\section{Discussion}

The aim of this study was to compare the outcomes of patients undergoing bariatric surgery over the years since introduction of the ERABS program in 2012. Since then, the ERABS protocol has continuously been evaluated and optimized.
In our previous analysis of the ERABS protocol as described by Mannaerts et al., the implementation of the program was mainly associated with logistic benefits, such as shorter operation time and shorter LOS [7]. Although the major complication rates remained stable, the number of hospital revisits had increased significantly. Under the hypothesis of this increase being caused by a gap in knowledge on the expected postoperative course, the ERABS protocol was adjusted. In the revised protocol, additional information-provided after surgery-concerning the postoperative diet and early mobilization with the physical therapist plays a key role. In the following years, significant decreases were seen in minor complications, readmissions and unplanned hospital revisits. Also, the duration of surgery decreased and the major complications rates remained stable. An important question that arises is whether these changes are caused by the revisions in the ERABS protocol, or that they are mainly influenced by the experience of the surgeon and the anesthesiological team.

The decrease in duration of surgery and LOS may partially be explained by the learning curve of the surgeon and anesthesiological team [13], but also by the effect of the ERABS protocol on the logistics around bariatric surgery [7]. Since 2016, the LOS remained stable. Patients are encouraged to leave the hospital on the first day 
postoperative, provided they meet the criteria for discharge according to the postoperative checklist. Nevertheless, hospital stay is prolonged on mild indications, to prevent premature discharge.

The decreasing minor complication rates and readmission rates are more likely to be caused by the improvements that were made to the ERABS protocol, as patients leave the hospital in optimal conditions: well informed and confident to go home for further recovery. Patients that did return to the hospital and/or were readmitted within 30 days postoperatively, more often actually had developed a complication, making the revisit or readmission justified. Mortality within 30 days has remained low with $0.05 \%$ annually over the years, which corresponds to the Dutch national average mortality rate of bariatric surgery of $0.05 \%$ [14].

With the finding of significantly less minor complications, hospital revisits and readmissions, this paper is the first ERABS paper to show an association with improvements in patient outcome rather than only logistic factors. While we aim for a further decrease in hospital revisits and readmissions, future research should focus on those patients who revisit the hospital without them having a complication. Also, future studies using questionnaires on Patient Reported Experience and Outcome Measures (PREMs/PROMs) may demonstrate an improvement in patient experience.

A limitation of this study is the variation in surgical experience between the surgeons. There are many factors that influence a surgeon's learning curve; the amount of performed bariatric procedures, the amount of other (laparoscopic) procedures performed and the number of bariatric procedures assisted, which can all have a substantial impact on their surgical skills. This study took place in a teaching hospital, meaning that the procedures were performed by bariatric surgeons or by residents under the supervision of a bariatric surgeon. Based on the number of performed procedures, we can stipulate that the five bariatric surgeons that performed the great majority of the procedures between 2014 and 2017 were in different stages of their learning curve. Even though their level of experience varied, the surgeon did not independently influence the complication rates in multivariate analysis. This result might be explained by the fact that we work with an experienced team of surgeons, scrub nurses and anesthesiology staff. Further research is required to determine the precise effect of surgical experience on patient outcome.

Our study underlines that the ERABS program is a dynamic concept and that it is important to continuously monitor and improve the ERABS protocol. This paper suggests that even minor alterations on dietary education and guided ambulation may already have a substantial impact on readmission rates. Besides the logistic benefits, ERABS also seems to improve patient outcome in terms of minor complications and readmissions within 30 days postoperatively. Smart timing of effective patient information provision seems to play an important role. In our opinion, optimization of the ERABS protocol is currently the main factor driving better outcomes. Further research is required to determine the impact of this improved ERABS programs on the patient's experience on the hospital admission, surgery and postoperative care. Optimization of analgesia, anti-emetics and the preoperative diet can be interesting topics for future research.

\section{Compliance with ethical standards}

Disclosures Drs. Leeman, van Mil, Biter, Apers, prof. Verhoef and Dr. Dunkelgrun have no conflicts of interest or financial ties to disclose.

Ethical approval All procedures performed in studies involving human participants were in accordance with the ethical standards of the institutional and/or national research committee and with the 1964 Helsinki declaration and its later amendments or comparable ethical standards. This study was approved by the Dutch medical ethical committee (METC).

Informed consent Informed consent was obtained from all individual participants included in the study.

\section{References}

1. Finucane MM, Stevens GA, Cowan MJ, Danaei G, Lin JK, Paciorek CJ et al (2011) National, regional, and global trends in body-mass index since 1980: systematic analysis of health examination surveys and epidemiological studies with 960 country-years and 9.1 million participants. Lancet (Lond, Engl). 377(9765):557-567

2. Neovius M, Narbro K, Keating C, Peltonen M, Sjoholm K, Agren $\mathrm{G}$ et al (2012) Health care use during 20 years following bariatric surgery. JAMA 308(11):1132-1141

3. Kehlet H (1997) Multimodal approach to control postoperative pathophysiology and rehabilitation. Br J Anaesth 78(5):606-617

4. Dogan K, Kraaij L, Aarts EO, Koehestanie P, Hammink E, van Laarhoven CJ et al (2015) Fast-track bariatric surgery improves perioperative care and logistics compared to conventional care. Obes Surg 25(1):28-35

5. Elliott JA, Patel VM, Kirresh A, Ashrafian H, Le Roux CW, Olbers T et al (2013) Fast-track laparoscopic bariatric surgery: a systematic review. Updat Surg 65(2):85-94

6. Lemanu DP, Singh PP, Berridge K, Burr M, Birch C, Babor $R$ et al (2013) Randomized clinical trial of enhanced recovery versus standard care after laparoscopic sleeve gastrectomy. $\mathrm{Br}$ J Surg 100(4):482-489

7. Mannaerts GH, van Mil SR, Stepaniak PS, Dunkelgrun M, de Quelerij M, Verbrugge SJ et al (2016) Results of implementing an enhanced recovery after bariatric surgery (ERABS) protocol. Obes Surg 26(2):303-312

8. Thorell A, MacCormick AD, Awad S, Reynolds N, Roulin D, Demartines $\mathrm{N}$ et al (2016) Guidelines for perioperative care in bariatric surgery: enhanced recovery after surgery (ERAS) society recommendations. World J Surg 40(9):2065-2083 
9. Malczak P, Pisarska M, Piotr M, Wysocki M, Budzynski A, Pedziwiatr M (2017) Enhanced recovery after bariatric surgery: systematic review and meta-analysis. Obes Surg 27(1):226-235

10. Brethauer SA, Kim J, El Chaar M, Papasavas P, Eisenberg D, Rogers A et al (2015) Standardized outcomes reporting in metabolic and bariatric surgery. Surg Obes Relat Dis 11(3):489-506

11. Fried M, Yumuk V, Oppert JM, Scopinaro N, Torres A, Weiner R et al (2014) Interdisciplinary European guidelines on metabolic and bariatric surgery. Obes Surg 24(1):42-55

12. van Mil SR, Duinhouwer LE, Mannaerts GHH, Biter LU, Dunkelgrun M, Apers JA (2017) The standardized postoperative checklist for bariatric surgery; a tool for safe early discharge? Obes Surg 27(12):3102-3109
13. Major P, Wysocki M, Dworak J, Pedziwiatr M, Pisarska M, Wierdak M et al (2018) Analysis of laparoscopic sleeve gastrectomy learning curve and its influence on procedure safety and perioperative complications. Obes Surg 28(6):1672-1680

14. Poelemeijer YQM, Liem RSL, Nienhuijs SW (2018) A Dutch nationwide bariatric quality registry: DATO. Obes Surg 28(6):1602-1610

Publisher's Note Springer Nature remains neutral with regard to jurisdictional claims in published maps and institutional affiliations. 